\title{
METABOTROPIC GLUTAMATE 5 RECEPTOR IN THE INFRALIMBIC CORTEX CONTRIBUTES TO DESCENDING PAIN FACILITATION IN HEALTHY AND ARTHRITIC ANIMALS
}

\author{
A. DAVID-PEREIRA, ${ }^{a, b}$ S. PUGA, ${ }^{a, b}$ S. GONÇALVES, ${ }^{a, b}$ \\ D. AMORIM, ${ }^{a, b}$ C. SILVA, ${ }^{a, b}$ A. PERTOVAARA, \\ A. ALMEIDA ${ }^{\mathrm{a}, \mathrm{b}}$ AND F. PINTO-RIBEIRO ${ }^{\mathrm{a}, \mathrm{b} *}$ \\ ${ }^{a}$ Life and Health Sciences Research Institute (ICVS), School \\ of Health Sciences (ECS), Campus of Gualtar, University of \\ Minho, 4750-057 Braga, Portugal \\ b ICVS/3B's - PT Government Associate Laboratory, \\ Braga/Guimarães, Portugal \\ ${ }^{\mathrm{c}}$ Biomedicum Helsinki, Institute of Biomedicine/Physiology, \\ University of Helsinki, Helsinki, Finland
}

\begin{abstract}
The involvement of the prefrontal cortex in pain processing has been recently addressed. We studied the role of the infralimbic cortex (IL) and group I metabotropic glutamate receptors (mGluRs) in descending modulation of nociception in control and monoarthritic (ARTH) conditions. Nociception was assessed using heat-induced paw withdrawal while drugs were microinjected in the IL of rats. Local anesthesia of the IL or the adjacent prelimbic cortex $(P L)$ facilitated nociception, indicating that IL and PL are tonically promoting spinal antinociception. Phasic activation with glutamate (GLU) revealed opposing roles of the PL and IL; GLU in the PL had a fast antinociceptive action, while in the IL it had a slow onset pronociceptive action. IL administration of a local anesthetic or GLU produced identical results in ARTH and control animals. An mGluR5 agonist in the IL induced a pronociceptive effect in both groups, while mGluR5 antagonists had no effect in controls but induced antinociception in ARTH rats. Activation of the IL mGluR1 (through co-administration of mGluR1/5 agonist and mGluR5 antagonist) did not alter nociception in controls but induced antinociception in ARTH animals. IL administration of an mGluR1 antagonist failed to alter nociception in either experimental group. Finally, mGluR5 but not mGluR1 antagonists blocked the pronociceptive action
\end{abstract}

\footnotetext{
${ }^{*}$ Correspondence to: F. Pinto-Ribeiro, Life and Health Sciences Research Institute (ICVS), School of Health Sciences (ECS), Campus of Gualtar, University of Minho, 4750-057 Braga, Portugal. Tel: + 351-253604852; fax: + 351-253604820.

E-mail address: filiparibeiro@ecsaude.uminho.pt (F. Pinto-Ribeiro). Abbreviations: ACC, anterior cingulate cortex; ARTH, K/C-induced monoarthritis; CHPG, (RS)-2-chloro-5-hydroxyglycine (mGluR5 agonist); DHPG, (S)-3,5-dihydroxyphenylglycine (mGluR1/5 agonist); GLU, glutamate; i.p., intraperitoneal; IL, infralimbic cortex; LIDO, lidocaine; LWT, limb withdrawal threshold; LY367385, (S)-(+)- $\alpha$-amino4-carboxy-2-methylbenzeneacetic acid (mGluR1 antagonist); mGluR, metabotropic glutamate receptor; MPEP, 6-methyl-2-(phenylethynyl) pyridine (mGluR5 antagonist); MTEP, 3-((2-methyl-1,3-thiazol-4-yl) ethynyl)pyridine hydrochloride (mGluR5 antagonist); mPFC, medial prefrontal cortex; PAM, pressure application measurement; PFC, prefrontal cortex; PL, prelimbic cortex; PWL, paw withdrawal latency; SHAM, control animals.
}

of GLU in both groups. The results indicate that IL contributes to descending modulation of nociception. mGluR5 in the IL enhance nociception in healthy control and monoarthritic animals, an effect that is tonic in ARTH. Moreover, activation of IL mGluR1s attenuates nociception following the development of monoarthritis. (c) 2015 IBRO. Published by Elsevier Ltd. All rights reserved.

Key words: infralimbic cortex, metabotropic glutamate receptor 5 , experimental monoarthritis, pronociception.

\section{INTRODUCTION}

In the last decade, there has been increasing evidence of the involvement of the prefrontal cortex (PFC) in the processing of the affective component of pain. It has been shown in both humans and animal models that, in chronic pain conditions, the PFC undergoes morphological and functional changes. These changes include decreased gray matter density in patients with chronic back pain (Apkarian et al., 2004). Increased medial PFC (mPFC) activation is correlated with the intensity and duration of spontaneous pain in patients with chronic pain (Baliki et al., 2006). Increases in the length, number of branches and spine density of basal dendrites of MPFC neurons as well as an increase in the NMDA/AMPA receptors ratio have been described in a rat model of neuropathic pain (Metz et al., 2009). Additionally, highfrequency electrical stimulation of the dorsal component of the MPFC, the anterior cingulate cortex (ACC), has been shown to be pronociceptive, decreasing heatevoked paw withdrawal latencies (Zhang et al., 2005). Less is known, however, of the pain modulatory role of the anatomically and functionally distinct ventral mPFC (Heidbreder and Groenewegen, 2003; Vertes, 2006) that consists of the prelimbic (PL) and infralimbic (IL) cortices in the rodent brain. Zhang et al. (2004) have evaluated the electrophysiological responses of mPFC neurons (ACC, $\mathrm{PL}$ and IL) to mechanical noxious stimulation of the rat's tail, and were able to distinguish two subsets of responding neurons (nociceptive specific and wide-dynamic range-like neurons) that seem to encode nociceptive stimulus intensity.

In the present work, we studied the contribution of the IL to the modulation of nociception in the rat. We used local cerebral microinjections to generally activate and 
inhibit the IL, in order to evaluate its role upon the descending modulation of nociceptive behavior of rats. Furthermore, we used specific receptor agonists and antagonists for group I glutamate metabotropic receptors (mGluRs; includes receptors 1 and 5 mGluR1 and mGluR5), in order to assess their involvement in IL-mediated descending modulation of nociception. The role of these receptors upon nociceptive modulation has been studied in several supraspinal brain areas (Palazzo et al., 2001; Neugebauer, 2002; Li and Neugebauer, 2004; Ren and Dubner, 2010), including the PL in the mPFC, where the blockade of mGluR1 can reverse the inhibition of neuronal spontaneous firing observed in sustained inflammatory pain (Ji and Neugebauer, 2011). Lastly, we investigated the impact of experimental monoarthritis upon IL-driven descending modulation of nociception.

\section{EXPERIMENTAL PROCEDURES}

\section{Animals, anesthetics and ethical issues}

The experiments were performed in adult Wistar Han male rats weighting 250-300 g (Charles River, France). The experimental protocol was approved by the Institutional Ethics Commission and followed the European Community Council Directive 2010/63/EU concerning the use of animals for scientific purposes. All efforts were made to minimize animal suffering and to use only the number of animals necessary to produce reliable scientific data.

During intracerebral cannula implantation, anesthesia was induced through the intraperitoneal (i.p.) administration of a mixture of ketamine $(0.75 \mathrm{mg} / \mathrm{kg}$, i.p.; Imalgene, Merial Lyon, France) and medetomidine $(0.5 \mathrm{mg} / \mathrm{kg}$, i.p.; Dorbene, Esteve Veterinaria, Léon, Spain). After the surgical procedures, anesthesia was reverted with atipamezole hydrochloride $(1 \mathrm{mg} / \mathrm{kg}$, i.p.; Antisedan, Orion Pharma, Orion Corporation, Espoo, Finland) and the animals were monitored until they were fully recovered. After the completion of the behavioral tasks, animals received a lethal dose of pentobarbitone and the brains were removed for histological confirmation of cannula placement (Fig. 1).

\section{Procedures for intracerebral injections}

For intracerebral drug administration, cannulae were implanted as described by Pinto-Ribeiro et al. (2011). Briefly, rats were placed in a stereotaxic frame, a longitudinal incision was made in the scalp, which was retracted as well as the subcutaneous fascia, and a sterilized stainless-steel guide cannula (26 gauge; Plastics One, Roanoke, VA, USA) was implanted in the brain through a hole drilled in the skull. The tip of the guide cannula was positioned $1 \mathrm{~mm}$ above the right IL or PL (as a placement control) using the following stereotaxic coordinates: IL: $2.76 \mathrm{~mm}$ frontal to bregma; $0.6 \mathrm{~mm}$ lateral to midline; depth $4.2 \mathrm{~mm}$; and PL: $2.76 \mathrm{~mm}$ frontal to the bregma; $0.6 \mathrm{~mm}$ lateral to midline; depth $3.5 \mathrm{~mm}$ (Paxinos and Watson, 2005). The guide cannula was fixed to the skull with screws and dental acrylic cement and the skin sutured around it. A dummy cannula (Plastics One) was inserted into the guide cannula to prevent contamination and the animals were allowed to recover from the surgery for at least one week.

Test drugs were administered through a 33-gauge injection cannula (Plastics One) protruding $1 \mathrm{~mm}$ beyond the tip of the guide cannula. The microinjection was performed using a $5.0 \mu \mathrm{L}$ Hamilton syringe connected to the injection cannula by a polyethylene catheter (PE-10; Plastics One). The injection volume was $0.5 \mu \mathrm{L}$ and therefore, the spread of the injected drugs within the brain was expected to have a diameter of $1 \mathrm{~mm}$ (Myers, 1966). The efficacy of the injection was monitored by observing the movement of a small air bubble through the tubing. The injection lasted at least $20 \mathrm{~s}$ and the injection cannula was left in place for additional $30 \mathrm{~s}$ to minimize the return of drug solution back to the injection cannula.

\section{Induction of monoarthritis}

The induction of a model of monoarthritis (ARTH) was performed 21 days before the beginning of the experiments, as described in detail elsewhere (PintoRibeiro et al., 2013). Briefly, 3\% kaolin and 3\% carrageenan (Sigma-Aldrich, St. Louis, MO, USA) were dissolved in distilled water and injected into the synovial cavity of the right knee joint at a volume of $0.1 \mathrm{~mL}$. This model produces mechanical hyperalgesia, which begins just in a few hours after surgery and extends up to 8 weeks (Radhakrishnan et al., 2003). In each animal, ARTH development was verified $1-2 \mathrm{~h}$ prior to each experiment. Only those rats that audibly vocalized every time after five flexion-extension movements of the knee joint were considered to have monoarthritis, and they were included in the ARTH group (Pinto-Ribeiro et al., 2011, 2013; Amorim et al., 2014). Control animals (SHAM) were injected with $0.1 \mathrm{~mL}$ saline in the synovial cavity of the right knee joint. SHAM animals did not vocalize to any of the five consecutive flexion-extension movements of the knee joint.

Additionally, we used the pressure application measurement (PAM) to verify the development of primary mechanical hyperalgesia in ARTH animals (Barton et al., 2007). To perform the test, the animal is held securely while the force transducer unit (fitted to the experimenter's thumb) is placed on one side of the knee joint and the forefinger on the other. Increasing force $(0-1500 \mathrm{~g})$ is gradually applied across the joint until a behavioral response is observed (paw-withdrawal, vocalization, wriggling or vocalization), with a cut-off of $5 \mathrm{~s}$. The peak force (in grams of force (gf)) applied immediately prior to the behavioral response is registered as the limb withdrawal threshold (LWT). LWT was measured twice in both the ipsilateral and contralateral limbs at 1-min intervals. The mean LWTs were calculated per animal. At the end of the session animals were returned to their home cage.

\section{Behavioral assessment of nociception - Hargreaves model}

Prior to performing the behavioral tests, rats were habituated to the experimental conditions (i) by allowing them to spend $1-2 \mathrm{~h}$ daily in the testing room during the week preceding any testing, and (ii) by performing daily 


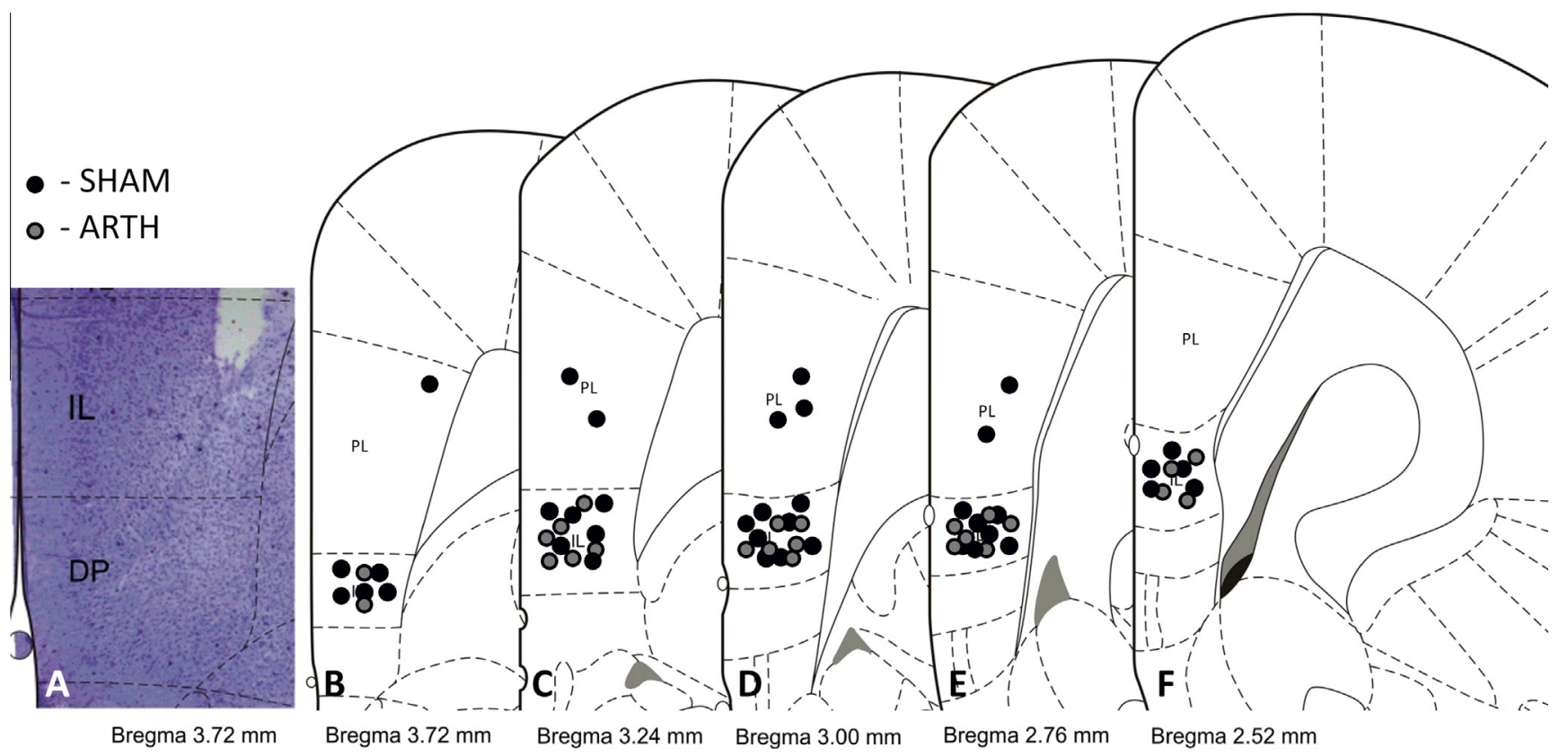

Fig. 1. Anatomical confirmation of drug injection sites in the prelimbic (PL) and infralimbic (IL) cortices. (A) Photomicrograph of an example of the drug injection site in the IL of the rat brain (AP: $+3.72 \mathrm{~mm}$ from bregma) superimposed with the appropriate legend of Paxinos and Watson (2005) stereotaxic atlas. (B-F) Schematic representation of other injection sites in the PL and IL $(B:+3.72 \mathrm{~mm}, \mathrm{C}:+3.24 \mathrm{~mm}, \mathrm{D}$ : $+3.00 \mathrm{~mm}, \mathrm{E}$ : $+2.76 \mathrm{~mm}$; F: $+2.52 \mathrm{~mm}$ ). DP - dorsal peduncular cortex; IL - infralimbic cortex; PL - prelimbic cortex.

handling sessions. For assessing nociception in unanesthetized animals, the latency of hindpaw withdrawal following radiant heat stimulation (Hargreaves test; Plantar Test Device Model 37370, Ugo Basile, Comerio, Italy) was determined. In each behavioral session, the withdrawal latency was assessed prior to drug administration and at various intervals following the intracerebral injections (Fig. 2). At each time point, the measurements were repeated twice at an interval of $1 \mathrm{~min}$ (except for glutamate (GLU) due to its fast effect) and the mean of these values was used in further calculations. The cut-off time for radiant-heat exposure was set at $15 \mathrm{~s}$ in order to avoid any damage to the skin.

\section{Drugs}

GLU (Merck, Darmstadt, Germany), (RS)-2-chloro-5hydroxyglycine (CHPG; mGluR5 agonist), (S)-3,5dihydroxyphenylglycine (DHPG; mGluR1/5 agonist; Tocris, Bristol, United Kingdom), and 3-((2-Methyl-1,3-thiazol-4-yl) ethynyl)pyridine hydrochloride (MTEP; mGluR5 antagonist, Tocris, Bristol, United Kingdom) solutions for intracerebral drug injection were prepared with sterilized saline solution 0.9\% (Unither, Amiens, France; pH 7,2). 6-Methyl-2(phenylethynyl)pyridine (MPEP; mGluR5 antagonist; Tocris, Bristol, United Kingdom) was dissolved in $10 \%$ dimethyl sulfoxide (DMSO). (S)-(+ )- $\alpha$-Amino-4-carboxy-2methylbenzeneacetic acid (LY367385; mGluR1 antagonist) was dissolved in $2 \%$ sodium hydroxide solution $(\mathrm{NaOH})$. Lidocaine (2\%; LIDO) was acquired as a solution (B. Braun Medical, Barcarena, Portugal).

Previous studies showed that the $50 \mathrm{nmol}$ dose of GLU (Pinto-Ribeiro et al., 2011), DHPG, CHPG (Ansah et al., 2009) and MPEP (Movsesyan et al., 2001) and the $40 \mathrm{nmol}$ dose of LY367385 (de Novellis et al., 2005) are effective in activating/blocking mGluRs after intracerebral microinjection in the rat. Since there are studies suggesting that MPEP has significant non-specific actions, including inhibition of NMDA receptors (Lea and Faden, 2006) and positive allosteric modulation of mGluR4 (Mathiesen et al., 2003), MTEP was also used and their effects compared. The MTEP dose $(50 \mathrm{nmol})$ used was the same as for MPEP to allow the comparison of the two antagonists' efficacy. An observation window of 50 min was determined by evaluating alterations in nociceptive behavior at fixed time points (Fig. 2) until the drug effect was no longer observed. Control injections with the respective vehicle (VEH) solutions were performed as control values, in order to avoid any bias that might result from injecting the solution itself.

\section{Rotarod test}

To exclude motor effects of drug injection in the IL, motor performance was evaluated on a Rotarod equipment (3376-4R; TSE Systems, USA) using an accelerating protocol. In this protocol, SHAM and ARTH animals were placed on a rod that accelerated smoothly from 4 to 40 rotations per minute (rpm) over a period of $5 \mathrm{~min}$. The first 3 days of protocol served as training. In each day, rats underwent the accelerating protocol for a total of 4 trials per day, with a rest of at least $20 \mathrm{~min}$ between each trial. On the following days, the effect of each drug upon motor performance was tested on the same accelerating protocol and the latency to fall was recorded (Monville et al., 2006). Due to the small window of drug action observed in the Hargreaves model, during drug testing animals underwent only two trials of the accelerating protocol, 10 and 30 min after drug administration (Fig. 2). 


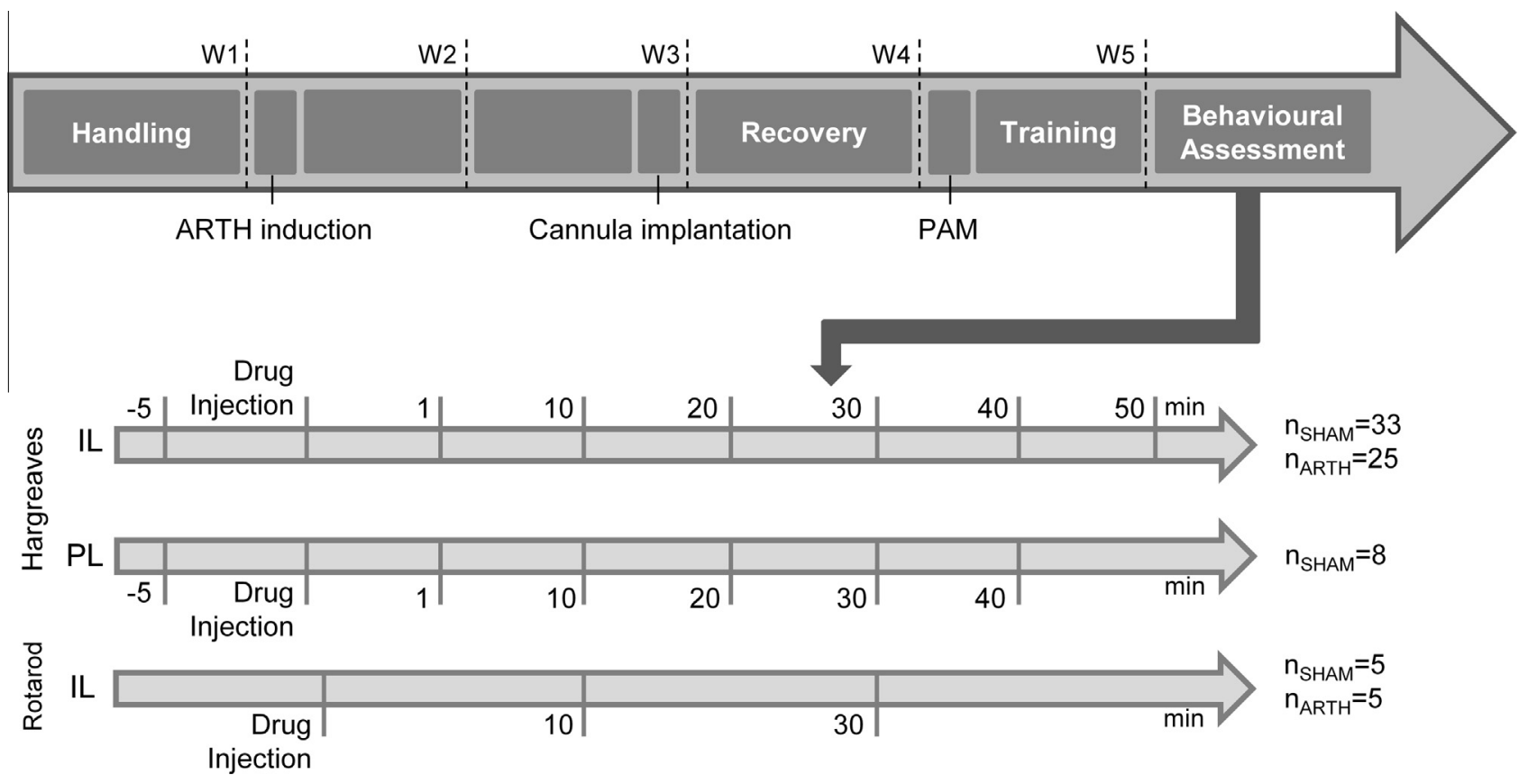

Fig. 2. Schematic representation of the experimental design timeline. Rats were habituated to the laboratory and the experimenter for 5 days. After habituation, animals belonging to the arthritic (ARTH) group received an intra-synovial injection of $3 \%$ kaolin/carrageenan while control (SHAM) animals received an intra-synovial injection of saline solution. Two weeks after monoarthritis induction, animals were implanted with a guide cannula in the infralimbic (IL) or in the prelimbic (PL) cortices. After recovery (one week), rats performed the pressure application measurement (PAM) and were trained in the paw-withdrawal apparatus. Pharmacological tests were performed at the same time points for all the drugs. min - minutes; W1-5 - weeks 1 to 5 .

\section{Course of the behavioral study}

Three weeks after ARTH induction and at least one week after guide cannula implantation, animals were trained in the Hargreaves test. Four weeks after ARTH induction, the tonic and phasic action of the IL and PL and the effect of the activation/inactivation of mGluR1 and/or mGluR5 in the IL upon nociceptive behavior were determined in unanesthetized animals through the assessment of changes in paw withdrawal latency (PWL) after drug injection. Withdrawal latencies were assessed 1, 10, 20, 30,40 and $50 \mathrm{~min}$ following intracerebral injections (Fig. 2). The interval between behavioral assessments of different drugs was of at least three days. The order for testing each different drug was randomized among animals. Animals were injected with a maximum of five different drugs, in random order.

\section{Statistics}

Using the GraphPad Prism 5 software (GraphPad Software Inc., La Jolla, CA, USA), a two-way analysis of variance (ANOVA) followed by $t$-test with a Bonferroni correction for multiple comparisons was used to compare behavioral results among experimental groups. $P<.05$ was considered to represent a significant difference. Data are presented as mean \pm standard error of the mean (SEM).

\section{RESULTS}

\section{Healthy animals}

GLU-induced activation of $P L$ and IL has opposite phasic effects on nociceptive behavior in healthy rats. In order to study a possible phasic role of the PL and the IL upon nociceptive behavior in healthy rats, we microinjected GLU into these areas and evaluated changes in the heat-evoked PWL of SHAM animals. Overall, GLU administration in the $\mathrm{PL}$ had an antinociceptive effect in SHAM animals, as revealed by an increase in the PWL (main effect of GLU: $\left.F_{1,120}=4.99 ; P=0.0273\right)$, and this effect varied with time (interaction drug effect $\times$ time: $F_{5,120}=2.36$, $P=0.0415)$. Post hoc tests showed that GLU treatment of the PL induced a short-lasting antinociceptive effect that was maximal $1 \mathrm{~min}$ after GLU injection and that disappeared within $10 \mathrm{~min}$ (Fig. 3A). In contrast, GLU administration in the IL resulted in a pronociceptive effect as revealed by the decrease of the PWL in SHAM animals (main effect of GLU: $F_{1,40}=15.73 ; P=0.0003$ ), and this pronociceptive effect varied with time (interaction drug $\times$ time: $\quad F_{5,40}=3.88, \quad P=0.0059$ ). While the antinociceptive effect induced by GLU in the PL was of rapid onset and short duration, post hoc tests indicated that GLU in the IL induced a pronociceptive effect that was significant from 10 to $30 \mathrm{~min}$ after GLU injection (Fig. 3C).

Local anesthesia of both $P L$ and $I L$ reveals tonic antinociceptive effects in healthy rats. To evaluate a possible tonic role of the $\mathrm{PL}$ and the $\mathrm{IL}$ in the descending control of nociceptive behavior in healthy rats, we microinjected LIDO, a local anesthetic, and evaluated changes in heat-evoked PWL of SHAM animals. Overall, the inhibition of PL and IL with LIDO significantly decreased PWL of SHAM animals (main effect of LIDO in PL: $F_{1,103}=11.63, P=0.0009$; main effect of LIDO in IL: $F_{1,44}=7.80 ; P=0.0077$ ), showing 

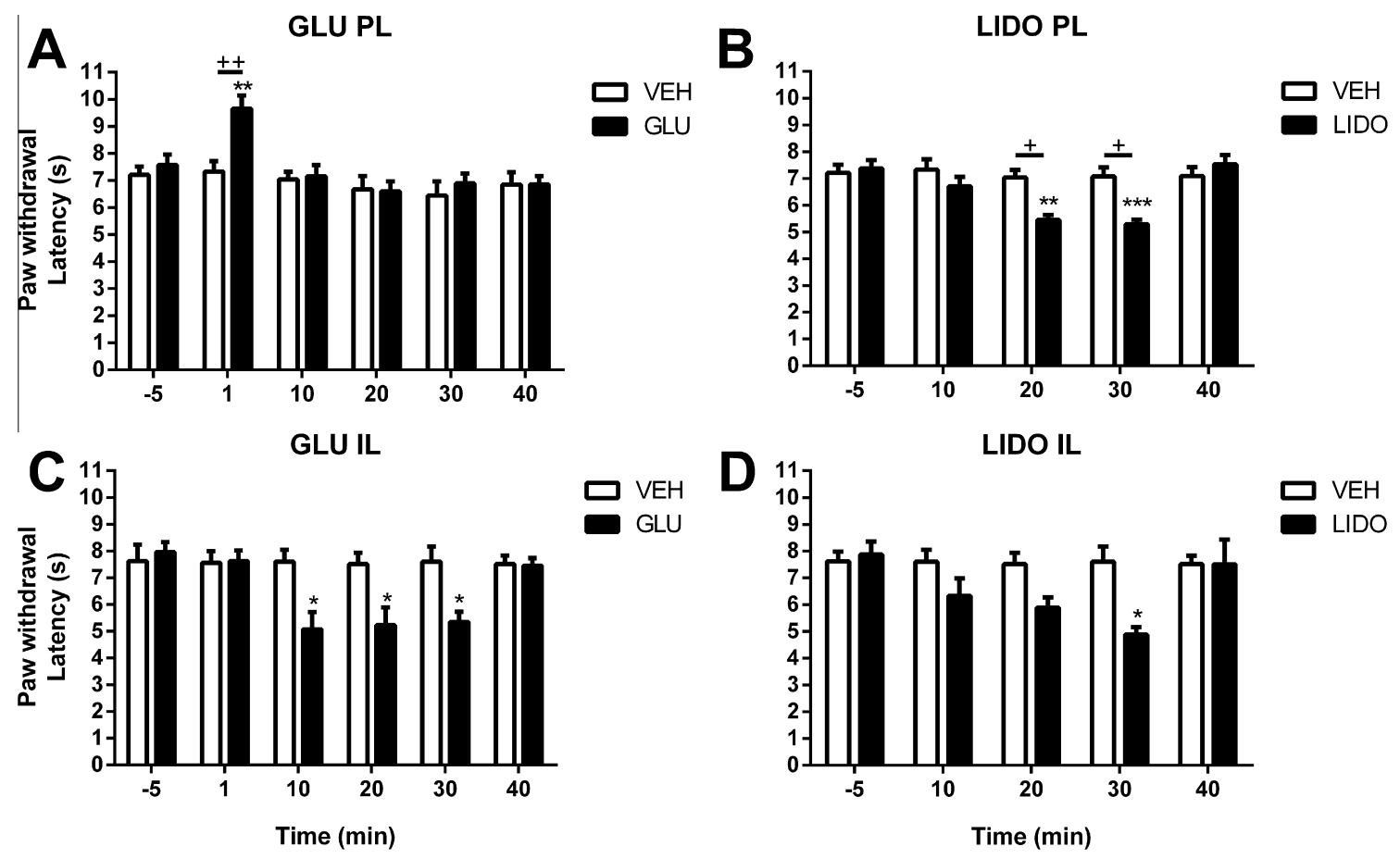

Fig. 3. Radiant heat-evoked paw withdrawal latencies $(P W L)$ of healthy control animals after intracerebral drug administrations in the prelimbic ( $P L$; A, B) or infralimbic (IL; C, D) cortex (GLU, glutamate, $50 \mathrm{nmol}$; LIDO, lidocaine, 2\%; VEH, vehicle). (A) GLU in the PL increased the PWL 1 min after its administration; (B) LIDO in the PL decreased the PWL 10 and 20 min after its administration; (C) GLU in the IL decreased the PWL 10-30 min after its administration; (D) LIDO in the IL decreased the PWL 30 min after its administration. Graphs show the mean + SEM $\left(V E H: n_{P L}=10\right.$; $n_{\mathrm{IL}}=6$; GLU: $n_{\mathrm{PL}}=10 ; n_{\mathrm{IL}}=6$; LIDO: $n_{\mathrm{PL}}=10 ; n_{\mathrm{IL}}=7$ ). Drug injections were performed at time point $0 .{ }^{* /+} P<0.05 ;{ }^{* *} /++P<0.01$; ${ }^{* \star \star} P<0.001$ ( $t$-test with a Bonferroni correction for multiple comparisons; ${ }^{* / * / / * * *}$ represent the comparison of injection results with pre-injection $(-5 \mathrm{~min})$ value; ${ }^{+/++}$represent the comparison of time point values of SHAM vs. ARTH).

that both these areas tonically inhibit nociception in healthy rats. Post-hoc tests showed that these LIDOinduced alterations in PWL lasted for 10-30 min (Fig. 3B, D).

The following experiments focused on the prolonged pronociceptive action of GLU in the IL.

\section{Effect of IL pharmacological manipulation upon nociceptive behavior}

The IL modulates heat-evoked nociceptive behavior of SHAM and ARTH animals. To assess if the long-lasting pronociceptive effect of GLU microinjection in the IL was due to activation of metabotropic rather than ionotropic receptors, we selectively activated mGluR1 and mGluR5 in the IL and assessed its impact upon nociceptive behavior of SHAM and ARTH animals. Additionally, we also determined the time window during which drugs microinjected into the IL affected nociceptive behavior by testing noxious heat-evoked PWL in SHAM and ARTH rats at various time points after cortical drug administrations (Figs. 4-6). IL injection of the VEH failed to alter PWL (main effect of time after $\mathrm{VEH}$ treatment: $F_{5,66}=0.142$; Fig. 4A), independent of the experimental group (interaction experimental group $\times$ time after vehicle treatment: $\left.F_{5,66}=0.05\right)$. GLU or LIDO in the IL significantly decreased PWL of SHAM and ARTH animals (main effect of time after GLU treatment: $F_{5,59}=14.80, P<0.0001$; main effect of time after LIDO treatment: $\left.F_{5,61}=8.70, P<0.0001\right)$ for $10-30$ min after drug injection (Figs. 4B, C). The pronociceptive effects of GLU or LIDO in the IL did not vary between the SHAM and ARTH groups (interaction experimental group $\times$ time after GLU microinjection: $F_{5,59}=0.48$; interaction experimental group $\times$ time after LIDO microinjection: $\left.F_{5,61}=0.70\right)$.

DHPG (an mGluR1/5 agonist) in IL significantly decreased PWL (main effect of time after DHPG treatment: $F_{5,66}=5.02, P=0.006$; Fig. 4D), an effect that varied with the experimental group (interaction experimental group $\times$ time after cortical drug treatment: $\left.F_{5,66}=3.76, P=0.0047\right)$. Post hoc tests indicated that the pronociceptive effect of DHPG in IL was significantly stronger in SHAM than ARTH animals 30 min after drug treatment (Fig. 4C).

Prolonged pronociceptive behavior elicited by GLU in the $I L$ is not mediated by mGluR1 activation. To assess if the mGluR1 was responsible for the long-lasting pronociceptive effect of GLU microinjection in the IL, we selectively activated and/or inhibited mGluR1 in the IL and assessed its impact on nociceptive behavior in SHAM and ARTH animals.

The IL co-administration of DHPG with MPEP (with the purpose of activating mGluR1) had a significant effect on PWL (main effect of time after DHPG + MPEP treatment: $\left.F_{5,74}=4.07, P=0.0026\right)$, that varied with the experimental group (interaction experimental group $\times$ time after drug treatment: $\left.F_{5,75}=7.96, P<0.0001\right)$. Post hoc tests indicated that the combination of DHPG 
VEH
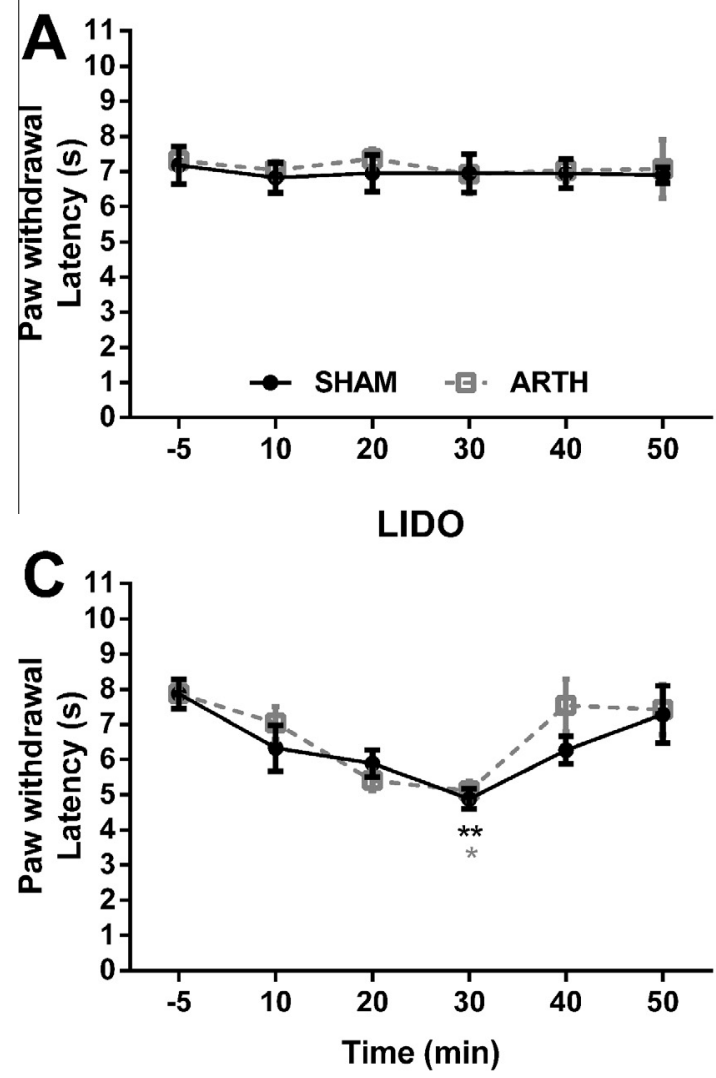

GLU

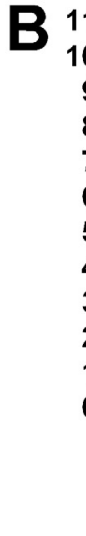

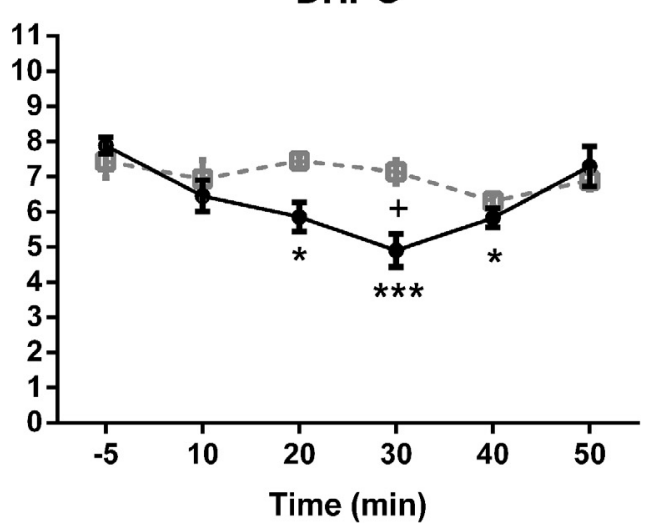

Fig. 4. Radiant heat-evoked paw withdrawal latencies (PWL) after intracerebral administration in the infralimbic cortex (IL). Effects of IL administration of vehicle (VEH; A), glutamate (GLU, $50 \mathrm{nmol}$; B), LIDO ( $\%$; C) and DHPG (an mGluR1/5 agonist, $50 \mathrm{nmol}$; D) in control (SHAM, black full lines) and monoarthritic (ARTH, gray dashed lines) animals. Drug injections were performed at time point 0 . Data are presented as mean + SEM. VEH: $n_{\mathrm{SHAM}}=6, n_{\mathrm{ARTH}}=8$; GLU: $n_{\mathrm{SHAM}}=6, n_{\mathrm{ARTH}}=6$; LIDO: $n_{\mathrm{SHAM}}=7, n_{\mathrm{ARTH}}=6 ; \mathrm{DHPG}: n_{\mathrm{SHAM}}=7, n_{\mathrm{ARTH}}=6 .{ }^{* /+} P<0.05$; ${ }^{* *} P<0.01 ;{ }^{* \star *} P<0.001$ ( $t$-test with a Bonferroni correction for multiple comparisons; ${ }^{* * * / * \star *}$ represent the comparison of injection results with preinjection $(-5 \mathrm{~min})$ value; ${ }^{+}$represents the comparison of time point values of SHAM vs. ARTH).

and MPEP prolonged the PWL only in the ARTH group and this antinociceptive effect was significantly stronger in the SHAM than the ARTH group from 10 to 30 min after the drug treatment (Fig. 5B). LY367385 alone (an mGluR1 antagonist) did not alter PWL (main effect of time after LY367385 treatment: $\quad F_{5,60}=0.4909, \quad P=0.7818$; Fig. 5C), independent of the experimental group (interaction experimental group $\times$ time after drug treatment: $\left.F_{5,60}=0.16\right)$. Co-administration of DHPG, MPEP and LY367385 in the IL failed to alter PWL (main effect of time after DHPG + MPEP + LY367358 treatment: $F_{5,60}=0.26, P=0.9321$ ), independent of the experimental group (interaction experimental group $\times$ time after drug treatment: $F_{5,60}=0.70$; Fig. 5D).

IL co-administration of LY367385 and GLU significantly decreased PWL of SHAM and ARTH animals (main effect of time after LY367358 + GLU treatment: $F_{5,128}=25.60, P<0.0001$ ) for $20-30 \mathrm{~min}$ after drug injection (Fig. 5E). The pronociceptive effects of the combination of LY367385 and GLU in the IL did not vary between SHAM and ARTH groups (interaction experimental group $\times$ time after microinjection: $\left.F_{5,128}=0.94\right)$.

No changes were observed in PWL after vehicle microinjection to the IL (main effect of time after VEH treatment: $\left.\quad F_{5,66}=0.14\right)$ independent of the experimental group (interaction experimental group $\times$ time after drug treatment: $F_{5,66}=0.05$; Fig. 5A).

mGluR5 mediates the prolonged pronociceptive behavior elicited by GLU in the IL. To assess if the long-lasting pronociceptive effect of GLU microinjection was mediated through mGluR5 in the IL, we selectively activated and/or inhibited mGluR5 in the IL and assessed its impact on nociceptive behavior in SHAM and ARTH animals.

CHPG (an mGluR5 agonist) in the IL significantly decreased PWL of SHAM and ARTH animals (main effect of time after CHPG treatment: $F_{5,120}=16,38$, $P<0.0001$ ) for 10-40 min after drug injection (Fig. 6B). The pronociceptive effects of CHPG in the IL did not vary between the SHAM and ARTH groups (interaction experimental group $\times$ time after microinjection: $\left.F_{5,120}=0.30\right)$. IL administration of MPEP or MTEP alone (mGluR5 antagonists) had a significant effect on PWL (main effect of time after MPEP treatment: $F_{5,82}=4.56$; $P<0.0001$; main effect of time after MTEP treatment: $\left.F_{5,77}=5.02 ; P=0.0005\right)$. The effect of MPEP or MTEP alone in IL varied with the experimental group (interaction experimental group $\times$ time after MPEP administration: $F_{5,82}=4.56, \quad P<0.0001 ; \quad$ interaction experimental 
VEH

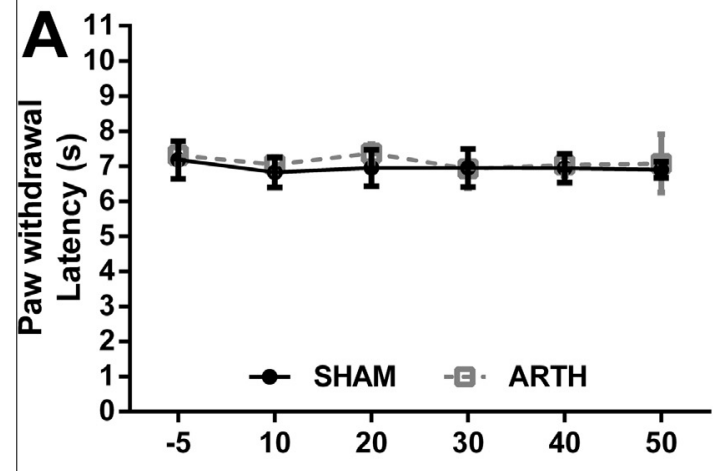

LY367385

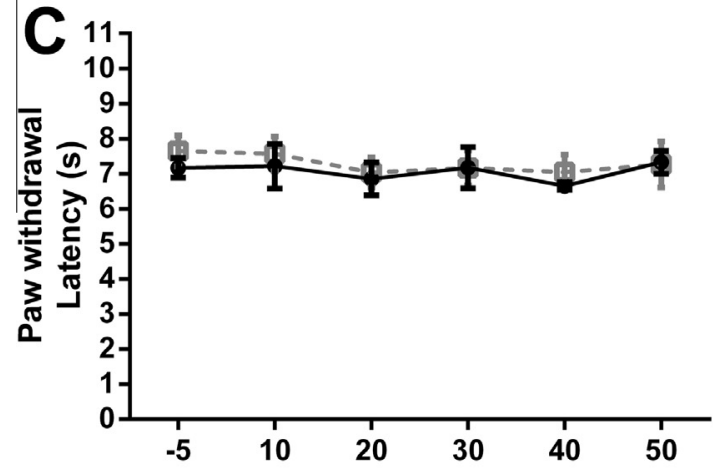

LY367385+GLU

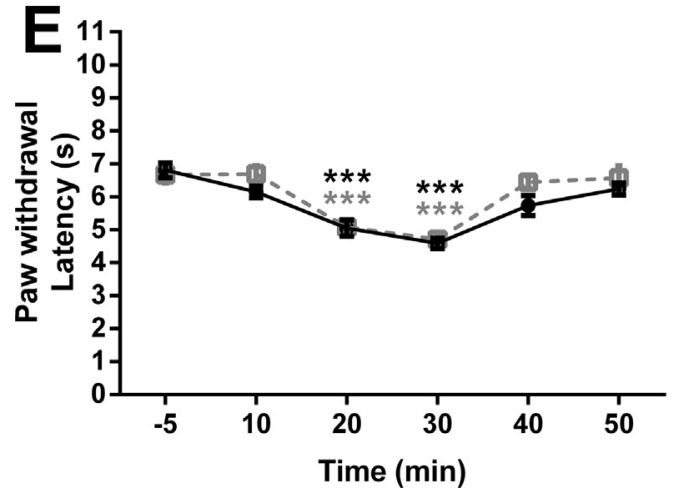

DHPG+MPEP

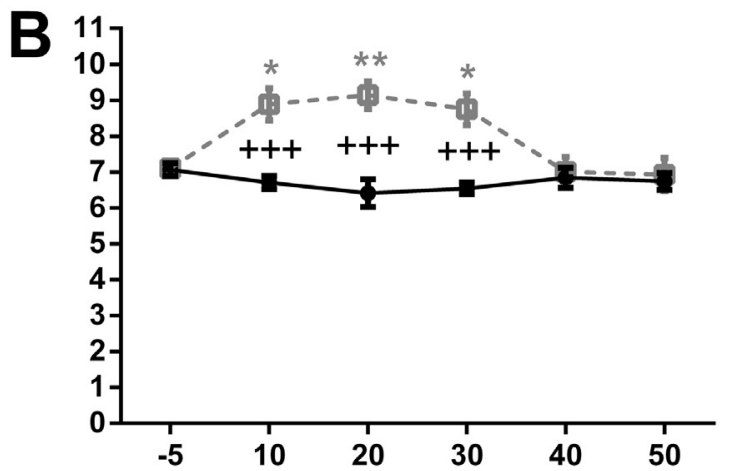

DHPG+MPEP+LY367385

D 11

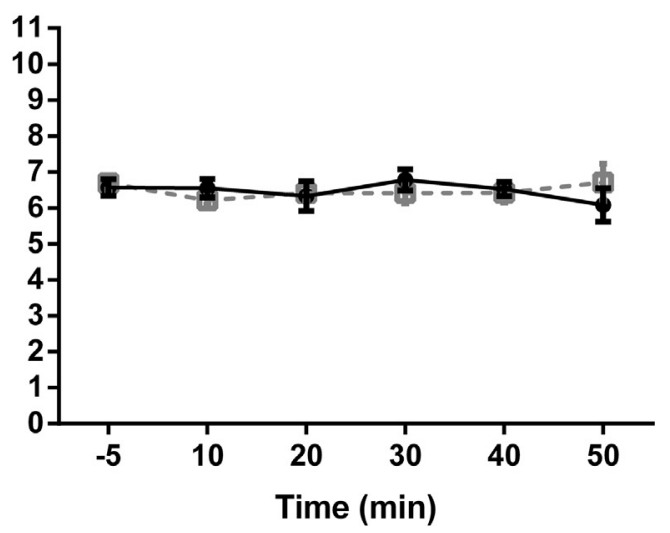

Fig. 5. Radiant heat-evoked paw withdrawal latencies (PWL) after intracerebral mGluR1 agonists/antagonists administration in the infralimbic cortex (IL). Effects of IL administration of vehicle (VEH; A), a combination of DHPG and MPEP (an mGluR1/5 agonist and an mGluR5 antagonist, respectively; $50 \mathrm{nmol}$ each; B), LY367385 (an mGluR1 antagonist, $40 \mathrm{nmol}$; C), a combination of DHPG, MPEP and LY367385 (50 nmol DHPG and MPEP, $40 \mathrm{nmol}$ LY367385; D) and a combination of LY367385 and GLU (40 nmol LY367385, $50 \mathrm{nmol}$ GLU; E) in control (SHAM, black full lines) and monoarthritic (ARTH, gray dashed lines) animals. Drug injections were performed at time point 0 . Data are presented as mean + SEM. VEH: $n_{\text {SHAM }}=6, n_{\text {ARTH }}=8 ;$ DHPG + MPEP: $n_{\text {SHAM }}=9, n_{\text {ARTH }}=6 ;$ LY367385: $n_{\text {SHAM }}=6, n_{\text {ARTH }}=6 ;$ DHPG + MPEP + LY367385: $n_{\text {SHAM }}=6$, $n_{\text {ARTH }}=6 ;$ LY367385 + GLU: $n_{\text {SHAM }}=11, n_{\text {ARTH }}=11 .{ }^{*} P<0.05 ;{ }^{* *} P<0.01 ;{ }^{* * *} /+++P<0.001$ (t-test with a Bonferroni correction for multiple comparisons; ${ }^{* / *} / * * *$ represent the comparison of injection results with pre-injection $(-5 \mathrm{~min})$ value; ${ }^{+++}$represent the comparison of time point values of SHAM vs. ARTH).

group $\times$ time after MTEP administration: $F_{5,77}=4.56$, $P=0.0010)$. Post hoc tests indicated that PWL was prolonged after MPEP or MTEP in the ARTH but not in the SHAM group and that the PWL was significantly longer in the ARTH than the SHAM group 30-40 min after MPEP or MTEP administration (Figs. 6C, D).
Co-administration of MPEP/MTEP and GLU in the IL failed to alter PWL (main effect of time after MPEP + GLU treatment: $F_{5,95}=0.21 ; \quad P=0.9568$; main effect of time after MTEP + GLU treatment: $F_{5,66}=$ $0.25 ; P=0.9396)$, independent of the experimental group (interaction experimental group $\times$ time after 
VEH
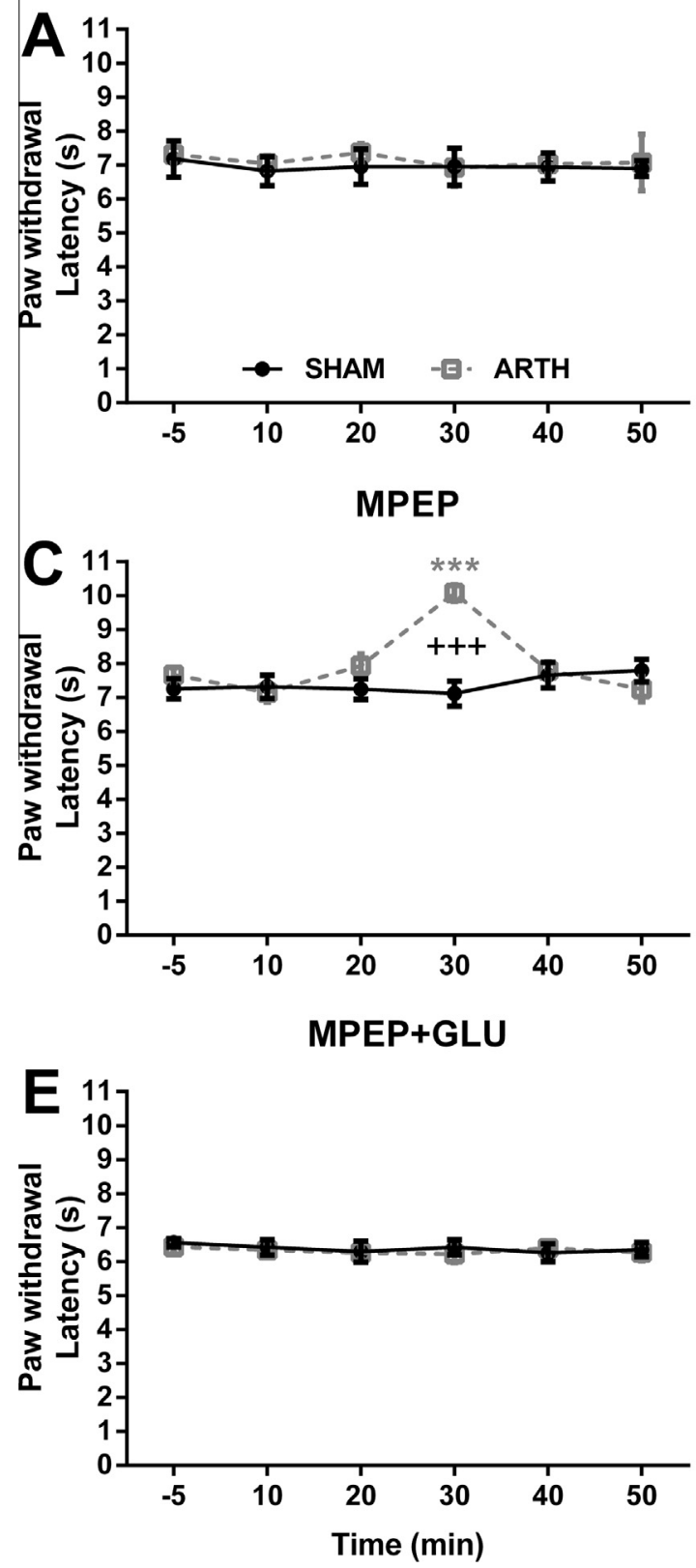

CHPG

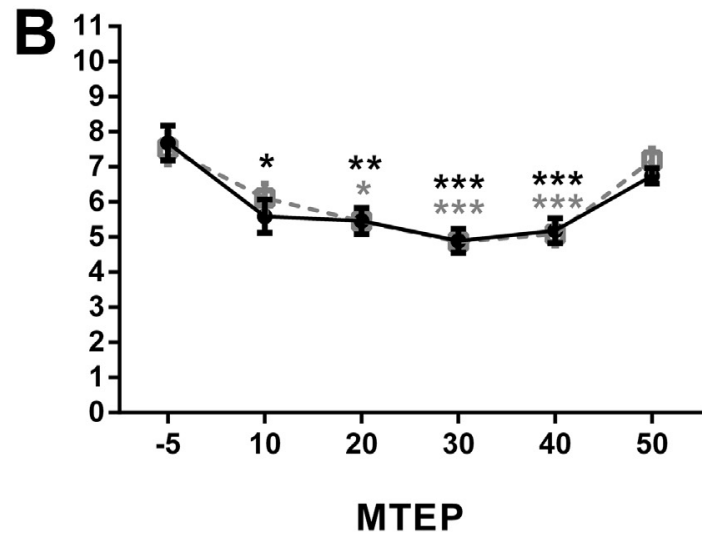

D 11

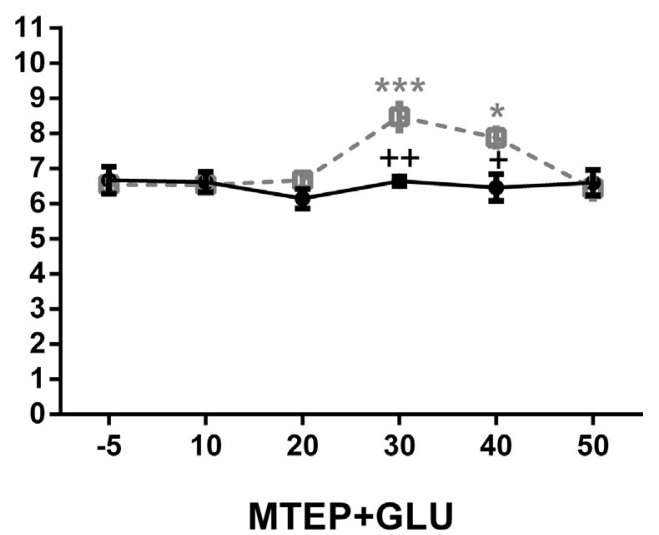

$\mathbf{F}$

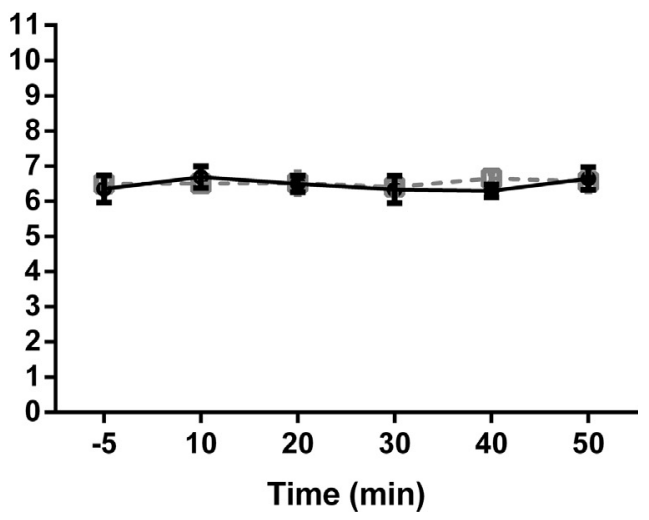

Fig. 6. Radiant heat-evoked paw withdrawal latencies (PWL) after intracerebral mGluR5 agonists/antagonists administration in the infralimbic cortex (IL). Effects of IL administration of vehicle (VEH; A), CHPG (an mGluR5 agonist, 50 nmol; B), MPEP (an mGluR5 antagonist, 50 nmol; C), MTEP (an mGluR5 receptor antagonist, $50 \mathrm{nmol}$; D), MPEP + GLU (50 nmol each; E) and MTEP + GLU (50 nmol each; F) in control (SHAM, black full lines) and monoarthritic (ARTH, gray dashed lines) animals. Drug injections were performed at time point 0 . Data are presented as mean + SEM. VEH: $n_{\text {SHAM }}=6, n_{\mathrm{ARTH}}=8 ;$ CHPG: $n_{\mathrm{SHAM}}=11, n_{\mathrm{ARTH}}=11 ; \mathrm{MPEP}: n_{\mathrm{SHAM}}=7, n_{\mathrm{ARTH}}=9 ; \mathrm{MTEP}: n_{\mathrm{SHAM}}=6, n_{\mathrm{ARTH}}=9 ; \mathrm{MPEP}$ + GLU: $n_{\mathrm{SHAM}}=11, n_{\mathrm{ARTH}}=7$; MTEP + GLU: $n_{\mathrm{SHAM}}=6, n_{\mathrm{ARTH}}=7 .{ }^{\star} /+P<0.05 ;{ }^{\star \star} /++{ }^{+} P<0.01 ;{ }^{\star \star *} /+++{ }^{+} P<0.001(t$-test with a Bonferroni

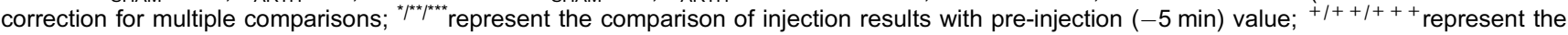
comparison of time point values of SHAM vs. ARTH).

MPEP + GLU treatment: $F_{5,95}=0.11$; interaction experimental group $\times$ time after MTEP + GLU treatment: $F_{5,66}=0.26$; Figs. 6E, F).

No changes were observed in PWL after vehicle microinjection to the IL (main effect of time after VEH treatment: $\left.F_{5,66}=0.14\right)$, independent of the experimental group (interaction experimental group $\times$ time after drug treatment: $F_{5,66}=0.05$; Fig. $6 \mathrm{~A}$ ).
ARTH animals present mechanical hyperalgesia in the affected knee joint. Four weeks after ARTH induction, mechanically evoked LWT of the knee joint of ARTH animals was significantly decreased when compared to SHAM (main effect of experimental group: $F_{1,26}=6.50$; $P=0.0171$ ), an effect dependent of the tested limb (interaction: experimental group $\times$ limb: $F_{1,26}=12.53$; $P=0.0015)$. Post hoc tests indicate that LWT in the 
ipsilateral joint of ARTH animals is significantly decreased when compared to the contralateral knee joint of the ARTH group and to the ipsilateral knee joint of SHAM group (Fig. 7A).

Motor performance was not altered after drug microinjection in the IL. Locomotor performance was assessed in the Rotarod test to evaluate potential motor effects elicited by drug administration. The results obtained show that although ARTH animals have a significantly decreased latency to fall when compared to the SHAM group (main effect of experimental group: $F_{1,64}=6.39$, $P=0.0140$ ), none of the microinjected drugs had an effect on motor performance of SHAM and ARTH rats at the time points tested in the nociceptive assessment (main effect of drug treatment: $F_{7,64}=0.10, P=$ 0.9980). This effect was independent of the experimental group (interaction experimental group $\times$ drug treatment: $F_{7,64}=0.12$; Fig. $7 \mathrm{~B}$ ) .

\section{DISCUSSION}

In the present work, we demonstrate for the first time that administration of GLU to the IL induces prolonged behavioral hyperalgesia. This effect is mediated by the mGluR5, since IL administration of a selective mGluR5 agonist mimicked the behavioral pronociceptive effect evoked by GLU in both SHAM and ARTH animals. Moreover, previous administration of an antagonist of mGluR5, but not mGluR1, in the IL was effective in blocking the pronociceptive effect of GLU in both experimental groups. The increase in withdrawal latency (antinociception) observed after blocking IL mGluR5 in ARTH animals only, suggests an increased tonic activation of these receptors in chronic inflammation of the joint.

The effect induced by activation of mGluR1 in the IL was studied indirectly by IL co-administration of an mGluR1/5 agonist and an mGluR5 antagonist. The antinociceptive effect induced by this combination of drugs in ARTH but not in SHAM animals suggests that following the development of monoarthritis, the net effect of the descending pathways recruited by mGluR1 is antinociceptive. It might be argued that the antinociception induced by IL co-administration of the mGluR1/5 agonist and mGluR5 antagonist in the ARTH group was due to blocking of the mGluR5-driven pronociceptive drive rather than activation of the mGluR1; however, previous administration of an mGluR1 antagonist blocked this antinociceptive effect, indicating an activation of mGluR1 instead of the inactivation of mGluR5. Additionally, the findings that IL administration of an mGluR5 agonist alone had a pronociceptive action whereas the mGluR $1 / 5$ agonist alone failed to alter nociception, support the proposal that mGluR1 in the IL of ARTH animals has indeed an antinociceptive effect.

\section{Technical considerations}

In this work, we have chosen to evaluate heat-evoked PWL, a test in which the baseline values of SHAM and
ARTH animals are similar (Fig. 4A), instead of mechanical LWT, where ARTH animals have significantly decreased values when compared to SHAM (Fig. 7A). Our choice was based on the technical differences between the PAM and the Hargreaves tests. The PAM test requires not only that the animals are heavily handled by the researcher during each experimental session, but also that the knee joint is noxiously stimulated twice at each time point before and after drug administration. Thus, one test would imply the affected joint to undergo 12 noxious stimulations in a short period of time $(60 \mathrm{~min})$ which by itself might bias the evaluation, as the mechanical hyperalgesia in $\mathrm{K} / \mathrm{C}$ model is use-dependent. By contrast, in the Hargreaves test animals are placed in a compartment for the duration of the experimental session (no handling is involved) and the thermal stimulus is applied from underneath the plantar surface of the hindpaw, thus sparing the joint, but still activating ascending and descending pain modulatory pathways. Therefore, we are not showing a reversion of mechanical hyperalgesia when treating ARTH animals, but that the IL promotes descending facilitation both in health and in disease, and that this effect is mediated by mGluR, mainly mGluR5.

\section{The opposing roles of the PL and IL in descending modulation of nociception are associated to the activation of different types of GLU receptors}

The dorsal portion of the MPFC, that includes the ACC, is among the most studied cortical areas in pain processing (Apkarian et al., 2005; Tracey and Mantyh, 2007), but only a few studies have been centered in the PL and IL cortices in the rodent brain. So far, these areas have been mostly implicated in the attentional and cognitive processing of pain (Apkarian et al., 2005), but there is some evidence that they actively modulate nociception. In fact, reports show that sustained pain conditions lead to a decrease of basal neuronal activity in the MPFC ( $\mathrm{Ji}$ and Neugebauer, 2011; Luongo et al., 2013). The behavioral data of the present study shows that the PL and the IL modulate nociception and that the blockade of these regions with LIDO decreased PWL, suggesting a tonic antinociceptive role in pain control.

Since the PL and the IL are adjacent to one another, it could be argued that there is widespread diffusion of drugs, resulting in a simultaneous activation/inactivation of these areas due to drug spillage outside of the targeted area of administration. However, GLU administration to the PL and IL had opposite effects on heat-evoked PWL, increasing and decreasing withdrawal latencies, respectively. Interestingly, GLU in the PL increased withdrawal latencies within 30-60 s, a short onset of action typical of the activation of ionotropic GLU receptors. Indeed, Millecamps et al. (2007) reported that activation of NMDA receptors in PL induced analgesia. By contrast, GLU in the IL decreased PWL, but only 10-30 min after drug administration, a response typically associated with the activation of mGluRs. This hypothesis is supported firstly, by the decrease in PWL observed after the IL microinjection of 
PAM

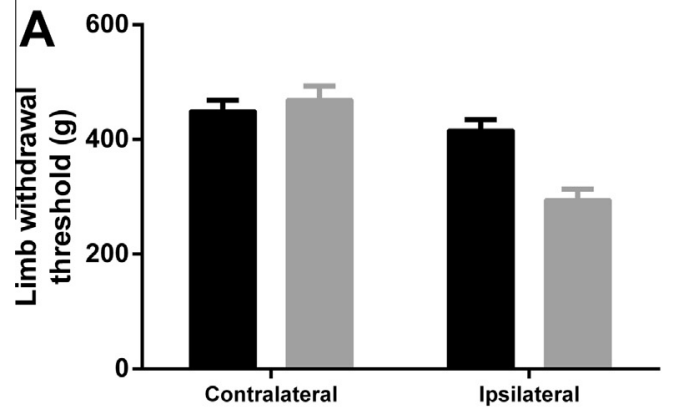

Rotarod

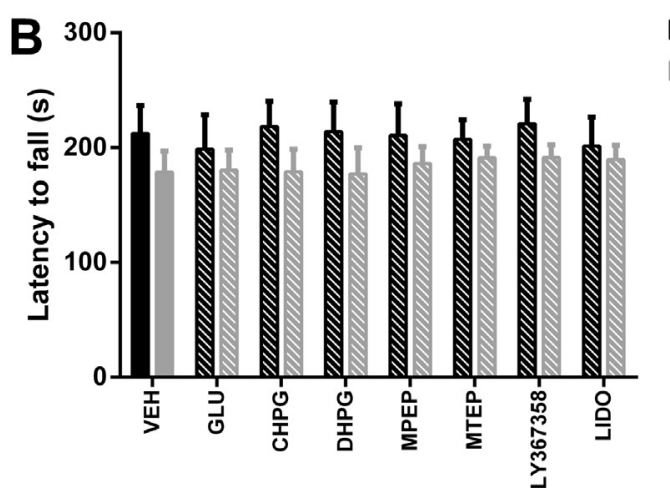

SHAM

ARTH

Fig. 7. (A) Evaluation of limb withdrawal threshold (LWT) in the pressure application measurement (PAM) 4 weeks after arthritis induction. The LWT of ARTH $(n=7)$ animals was significantly decreased when compared to results of control SHAM ( $n=8)$ animals (mechanical hyperalgesia). (B) Drug effects on locomotion. Evaluation of performance in the rotarod test after drug injection in the infralimbic cortex (IL) of SHAM and ARTH rats showed that none of the drugs had an effect on the latency to fall 10-30 min after administration in the IL. Data are presented as mean + SEM. ${ }^{\star \star} P<0.01 ;{ }^{\star \star \star} P<0.001$.

CHPG, an mGluR5 selective agonist, which mimicked the effect of GLU in the IL; and secondly, by the lack of changes in nociceptive behavior when GLU microinjection was preceded by administration of MPEP or MTEP, two different mGluR5 antagonists.

Interestingly, GLU and CHPG microinjection decreased PWL similar to what observed after LIDO microinjection. A potential explanation for this finding is that the effect of mGluR5 activation triggers an inhibitory mechanism, leading to suppression of neuronal discharge in the IL. In line with this hypothesis, a recent study by Pollard et al. (2014) has shown that mGluR5 activation leads to inhibition of neuronal activity in the ventral mPFC by promoting feed-forward inhibition. However, there are also contrasting reports that show mGluR5 activation in the ventral $\mathrm{MPFC}$ increases neuronal excitability by reducing the release of presynaptic GABA (Kiritoshi et al., 2013; Ji and Neugebauer, 2014). As a modulator of neuronal excitability (Schoepp, 2001), mGluR5 activation/inactivation can affect several mechanisms, thus, further studies are still needed to fully understand the pathways underlying descending modulation of nociception modulated by mGluR5 in the IL.

\section{mGluRs mediate GLU-driven descending facilitation from the IL}

In the present work, IL administration of the selective mGluR5 agonist CHPG as well as of exogenous GLU induced delayed and long-lasting pronociceptive effects that were identical in both SHAM and ARTH animals. Although the mechanism driving this effect is not fully understood, a study by Ji and Neugebauer (2014) showed the administration of an mGluR5-positive allosteric modulator (which increases receptor availability without activating it) increases background and evoked activity of IL pyramidal cells in healthy animals. However, in animals with sustained inflammatory pain, this facilitatory effect was only observed following co-application of a CB1 receptor agonist with the mGluR5 allosteric modulator (Ji and Neugebauer, 2014). This finding indicates that sustained inflammatory pain promotes remodeling of signaling pathways involving the IL and mGluRs. In line with this evidence, we observed that MPEP or MTEP, both mGluR5 antagonists, in the IL produced antinociception only in ARTH animals, further suggesting that mGluR5 in the IL plays a role in tonic facilitation of nociception in chronic inflammatory disorders. Other studies using animal models of inflammatory pain have reported antinociception (Hudson et al., 2002; Zhu et al., 2004) and inhibition of spontaneous burst activity in the mPFC (Houmayoun and Moghaddam, 2006) after systemic administration of an mGluR5 antagonist. Together, the effects observed after blocking mGluR5 suggest this receptor plays an important role in the modulation of nociceptive transmission in chronic inflammatory pain states. Although we cannot directly compare the effect of systemically administrated drugs to the effect of local microinjections in a specific brain area, the present and earlier results (Ji and Neugebauer, 2014) are in line with the proposal that the mGluR5-mediated mechanisms in the IL contribute to the descending control of nociception and its modulation in inflammatory conditions.

Activation of mGluR1 with a combination of DHPG and MPEP in the IL of SHAM animals had no effect upon nociceptive behavior, while it increased heatevoked PWL (antinociception) in the ARTH group. These results suggest that in experimental monoarthritis a descending antinociceptive pathway can be activated if mGluR1 in the IL are recruited. Yet, inactivation of mGluR1 in the IL with antagonist LY367385 had no effect on PWL of SHAM or ARTH animals, indicating that mGluR1 are not tonically activated in the IL. Interestingly, an earlier electrophysiological study showed PL mGluR1 are important players in the decrease of the spontaneous activity of PL neurons caused by pain-induced hyperactivity of the amygdala in sustained inflammatory conditions ( $\mathrm{Ji}$ and Neugebauer, 2014). Overall, although we were able to evoke an antinociceptive effect after the activation of mGluR1 in the IL, the impact of this pathway toward nociception remains unclear, since the blockade of mGluR1 had no 
effect upon nociceptive behavior and the pronociceptive effect of mGluR5 prevailed in controls and animals with experimental monoarthritis.

\section{CONCLUSIONS}

Drugs acting at mGluRs have more subtle effects on glutamatergic transmission than agonists and antagonists of ionotropic GLU receptors, as well as fewer side effects on normal functions (Conn and Pin, 1997; Schoepp, 2001; Neugebauer, 2002). Hence, the modulation of mGluRs allows a fine-tuning of cellular responses to glutamatergic inputs. The results of this study provide strong evidence the IL is involved in the descending modulation of nociception and mGluRs, particularly mGluR5, might contribute to inflammatory hyperalgesia.

\begin{abstract}
Acknowledgments-This study was supported by grants from the Portuguese Science Foundation (FCT) Project PTDC/SAUNEU/108557/2008, FEDER-COMPETE, by the Academy of Finland and the Sigrid Juselius Foundation, Helsinki, Finland and co-financed by the Portuguese North Regional Operational Program (ON.2 - O Novo Norte) under the National Strategic Reference Framework (QREN), through the European Regional Development Fund (FEDER). ADP was supported by FCT grant SFRH/BD/90374/2012 and DA was supported by FCT grant SFRH/BD/71219/2010.
\end{abstract}

\section{REFERENCES}

Amorim D, David-Pereira A, Pertovaara A, Almeida A, Pinto-Ribeiro F (2014) Amitriptyline reverses hyperalgesia and improves associated mood-like disorders in a model of experimental monoarthritis. Behav Brain Res 265:12-21.

Ansah OS, Gonçalves L, Almeida A, Pertovaara A (2009) Enhanced pronociception by amygdaloid group I metabotropic glutamate receptors in nerve-injured animals. Exp Neurol 216:66-74.

Apkarian AV, Bushnell MC, Treede RD, Zubieta JK (2005) Human brain mechanisms of pain perception and regulation in health and disease. Eur J Pain 9:463-484.

Apkarian AV, Sosa Y, Sonty S, Levy RM, Harden RN, Parrish TB, Gitelman DR (2004) Chronic back pain is associated with decreased prefrontal and thalamic gray matter density. J Neurosci 24:10410-10415.

Baliki MN, Chialvo DR, Geha PY, Levy RM, Harden RN, Parrish TB, Apkarian AV (2006) Chronic pain and emotional brain: specific brain activity associated with spontaneous fluctuations of intensity of chronic back pain. J Neurosci 26:12165-12173.

Barton NJ, Strickland IT, Bond SM, Brash HM, Bate ST, Wilson AW, Chessell IP, Reeve AJ, McQueen DS (2007) Pressure application measurement (PAM): a novel behavioural technique for measuring hypersensitivity in a rat model of joint pain. J Neurosci Methods 163:67-75.

Conn PJ, Pin JP (1997) Pharmacology and functions of metabotropic glutamate receptors. Annu Rev Pharmacol Toxicol 37:205-237.

de Novellis V, Mariani L, Palazzo E, Vita D, Marabese I, Scafuro M, Rossi F, Maione S (2005) Periaqueductal grey CB1 cannabinoid and metabotropic glutamate subtype 5 receptors modulate changes in rostral ventromedial medulla neuronal activities induced by subcutaneous formalin in the rat. Neuroscience 134:269-281.

Heidbreder CA, Groenewegen HJ (2003) The medial prefrontal cortex in the rat: evidence for a dorso-ventral distinction based upon functional and anatomical characteristics. Neurosci Biobehav Rev 27:555-579.
Houmayoun H, Moghaddam B (2006) Bursting of prefrontal cortex neurons in awake rats is regulated by metabotropic glutamate 5 (mGlu5) receptors: rate-dependent influence and interaction with NMDA receptors. Cereb Cortex 16:93-105.

Hudson LJ, Bevan S, McNair K, Gentry C, Fox A, Kuhn R, Winter J (2002) Metabotropic glutamate receptor 5 upregulation in A-fibers after spinal nerve injury: 2-methyl-6-(phenylethynyl)-pyridine (MPEP) reverses the induced thermal hyperalgesia. J Neurosci 22:2660-2668.

Ji G, Neugebauer V (2011) Pain-related deactivation of medial prefrontal cortical neurons involves mGluR1 and $\mathrm{GABA}_{A}$ receptors. J Neurophysiol 106:2642-2652.

Ji G, Neugebauer V (2014) CB1 augments mGluR5 function in medial prefrontal cortical neurons to inhibit amygdala hyperactivity in an arthritis pain model. Eur J Neurosci 39:455-466.

Kiritoshi T, Sun H, Ren W, Stauffer SR, Lindsley CW, Conn PJ, Neugebauer V (2013) Modulation of pyramidal cell output in the medial prefrontal cortex by mGluR5 interacting with CB1. Neuropharmacology 66:170-178.

Li W, Neugebauer V (2004) Differential roles of mGluR1 and mGluR5 in brief and prolonged nociceptive processing in central amygdala neurons. J Neurophysiol 91:13-24.

Lea PMT, Faden Al (2006) Metabotropic glutamate receptor subtype 5 antagonists MPEP and MTEP. CNS Drug Rev 12:149-166.

Luongo L, de Novellis V, Gatta L, Palazzo E, Vita D, Guida F, Giordano C, Siniscalco D, Marabese I, De Chiaro M, Boccella S, Rossi F, Maione S (2013) Role of metabotropic glutamate receptor 1 in the basolateral amygdala-driven prefrontal cortical deactivation in inflammatory pain in the rat. Neuropharmacology 66:317-329.

Mathiesen JM, Svendsen N, Brauner-Osborne H, Thomsen C, Ramirez MT (2003) Positive allosteric modulation of the human metabotropic glutamate receptor 4 (hmGluR4) by SIB-1893 and MPEP. Br J Pharmacol 138:1026-1030.

Metz AE, Yau HJ, Centeno MV, Apkarian AV, Martina M (2009) Morphological and functional reorganization of rat medial prefrontal cortex in neuropathic pain. Proc Natl Acad Sci USA 106:2423-2428.

Millecamps M, Centeno MV, Berra HH, Rudick CN, Lavarello S, Tkatch T, Apkarian AV (2007) D-Cycloserine reduces neuropathic pain behavior through limbic NMDA-mediated circuitry. Pain 132:108-123.

Monville C, Torres EM, Dunnett SB (2006) Comparison of incremental and accelerating protocols of the rotarod test for the assessment of motor deficits in the 6-OHDA model. J Neurosci Methods 158:219-223.

Movsesyan VA, O'Leary DM, Fan L, Bao W, Mullins PGM, Knoblach SM, Faden Al (2001) MGluR5 antagonists 2-methyl-6(phenylethynyl)-pyridine and (E)-2-methyl-6-(2-phenylethenyl)pyridine reduce traumatic neuronal injury in vitro and in vivo by antagonizing $\mathrm{N}$-methyl-D-aspartate receptors. J Pharmacol Exp Ther 296:41-47.

Myers RD (1966) Injection of solutions into cerebral tissue: relation between volume and diffusion. Physiol Behav 1:171-174.

Neugebauer V (2002) Metabotropic glutamate receptors - important modulators of nociception and pain behaviour. Pain 98:1-8.

Palazzo E, Marabese I, de Novellis V, Oliva P, Rossi F, Berrino L, Rossi F, Maione S (2001) Metabotropic and NMDA glutamate receptors participate in the cannabinoid-induced antinociception. Neuropharmacology 40:319-326.

Paxinos G, Watson C (2005) The rat brain in stereotaxic coordinates. Sydney: Academic Press.

Pinto-Ribeiro F, Amorim D, David-Pereira A, Monteiro AM, Costa P, Pertovaara A, Almeida A (2013) Pronociception from the dorsomedial nucleus of the hypothalamus is mediated by the rostral ventromedial medulla in healthy controls but is absent in arthritic animals. Brain Res Bull 99:100-108.

Pinto-Ribeiro F, Ansah OB, Almeida A, Pertovaara A (2011) Response properties of nociceptive neurons in the caudal ventrolateral medulla (CVLM) in monoarthritic and healthy control rats: Modulation of responses by the paraventricular nucleus of the hypothalamus (PVN). Brain Res Bull 86:82-90. 
Pollard M, Bartolome JM, Conn PJ, Steckler T, Shaban H (2014) Modulation of neuronal microcircuit activities within the medial prefrontal cortex by mGluR5 positive allosteric modulator. J Psychopharmacol 28:935-946.

Radhakrishnan R, Moore SA, Sluka KA (2003) Unilateral carrageenan injection into muscle or joint chronic bilateral hyperalgesia in rats. Pain 104:567-577.

Ren K, Dubner R (2010) Glutamate and GABA receptors in pain transmission. In: Beaulieu P, Lussier D, Porreca F, Dickenson $\mathrm{AH}$, editors. Pharmacology of pain. Seattle: IASP Press, International Association for the Study of Pain. p. 207-239.

Schoepp DD (2001) Unveiling the functions of presynaptic metabotropic glutamate receptors in the central nervous system. J Pharmacol Exp Ther 299:12-20.

Tracey I, Mantyh PW (2007) The cerebral signature for pain perception and its modulation. Neuron 55:377-391.
Vertes RP (2006) Interactions among the medial prefrontal cortex, hippocampus and midline thalamus in emotional and cognitive processing in the rat. Neuroscience 142:1-20.

Zhang L, Zhang Y, Zhao ZQ (2005) Anterior cingulate cortex contributes to the descending facilitatory modulation of pain via dorsal reticular nucleus. Eur J Neurosci 22(5):1141-1148.

Zhang R, Tomida M, Katayama Y, Kawakami Y (2004) Response durations encode nociceptive stimulus intensity in the rat medial prefrontal cortex. Neuroscience 126:777-785.

Zhu CZ, Wilson SG, Mikusa JP, Wismer CT, Gauvin DM, Lynch 3rd JJ, Wade CL, Decker MW, Honore P (2004) Assessing the role of metabotropic glutamate receptor 5 in multiple nociceptive modalities. Eur J Pharmacol 506:107-118. 